\title{
Geometry-induced localization of thermal fluctuations in ultrathin superconducting structures
}

\author{
W. V. Pogosov, ${ }^{1,2}$ V. R. Misko, ${ }^{1}$ and F. M. Peeters ${ }^{1}$ \\ ${ }^{1}$ Departement Fysica, Universiteit Antwerpen, Groenenborgerlaan 171, B-2020 Antwerpen, Belgium \\ ${ }^{2}$ Institute for Theoretical and Applied Electrodynamics, Russian Academy of Sciences, Izhorskaya 13, 125412 Moscow, Russia
}

(Received 16 June 2010; published 27 August 2010)

\begin{abstract}
Thermal fluctuations of the order parameter in an ultrathin triangular-shaped superconducting structure are studied near $T_{c}$, in zero applied field. We find that the order parameter is prone to much larger fluctuations in the corners of the structure as compared to its interior. This geometry-induced localization of thermal fluctuations is attributed to the fact that condensate confinement in the corners is characterized by a lower effective dimensionality, which favors stronger fluctuations.
\end{abstract}

DOI: $10.1103 /$ PhysRevB.82.054523

PACS number(s): 74.25.Bt, 74.25.Uv, 74.78.Na

\section{INTRODUCTION}

Recent progress in growth technology has made it possible to fabricate ultrathin films, consisting of just few monoatomic layers. Very recently ${ }^{1}$ superconducting properties of single-atomic layer films made of $\mathrm{Pb}$ were reported, including even the observation of Abrikosov vortices. It is also possible to make nanostructures and microstructures based on such thin films. These nanostructures are generally of much higher quality than those, used in earlier experiments, ${ }^{2}$ which, in particular, were not completely free of pinning centers.

It is well known that the lower the dimensionality of the system the stronger the thermal fluctuations. Ultrathin superconducting films as well as nanostructures based on such films are obvious candidates for the observation of thermal fluctuations. Indeed, it was reported very recently that strong current-induced thermal fluctuations of the order parameter were observed in superconducting nanowires fabricated in a meander of $\mathrm{NbN}^{3}$ Structures of this kind are used in highly sensitive photodetectors. It turns out that the fluctuations are a main source of dark-count events in such photodetectors: this means that their effect is actually parasitic. Fluctuation phenomena were interpreted in terms of a thermally activated entry of vortices, as well as by unbinding of vortexantivortex pairs. ${ }^{3}$ In another recent experiment, ${ }^{4}$ no hysteresis for vortex penetration and expulsion was observed in the case of a nanoisland of $\mathrm{Pb}$, which was so small that it could accommodate only one vortex. In a similar experiment, ${ }^{5}$ performed at lower temperature, some hysteresis was detected but the width of the hysteresis region was significantly smaller than expected from theory. A theoretical explanation for these phenomena was very recently suggested by one of us in terms of a thermal suppression of the surface barrier for vortex entry/exit, which might occur in superconducting nanoislands made of $\mathrm{Pb}$ in the regime of the ultimate single vortex confinement. ${ }^{6}$ Previously, thermal activation of vortices over the surface barrier was demonstrated to be possible in high- $T_{c}$ low-dimensional structures. ${ }^{7}$

Geometry itself can play an important role in fluctuation phenomena. The shape of islands, prepared by the method of evaporation in ultrahigh vacuum, depends strongly on their sizes. They grow according to the Stranski-Krastanov scenario, i.e., starting from well-facetted nuclei. ${ }^{4}$ As a result, islands with lateral dimensions of nearly $100 \mathrm{~nm}$ and smaller have a hexagonal shape while larger islands tend to have a triangular shape [see, e.g., Fig. 1(a) in Ref. 4]. At the same time, it is known that the triangular geometry can lead to very peculiar consequences for the superconducting condensate confined in this geometry. For instance, it was shown in Refs. 8 and 9 that stable vortex-antivortex molecules can nucleate, when a homogeneous magnetic field is applied. The aim of the present paper is to explore how triangularity, or more generally the presence of sharp corners in a thin superconducting structure, influences thermal fluctuations of the order parameter. The motivation to study the triangular geometry, apart from possible links with experiments, is that one can expect thermal fluctuations to be stronger in the corners of a triangular structure as compared to its interior since the superconducting condensate in the corners is strongly confined by samples' borders. This implies that the system somehow is characterized by lower effective dimensionality than in the interior. It is however not evident $a$ priori if this rather general argument leads to any noticeable effect for the fluctuations of the order parameter in a triangle so that more careful inspection is certainly desirable. In order to reveal the effect of geometry on spatial localization of thermal fluctuations, we restrict ourselves to the case of zero applied field but we consider temperatures both below and above $T_{c}$. We apply the method of small-amplitude oscillations within the Ginzburg-Landau theory, and we do not consider the possible excitation of vortices.

The paper is organized as follows. In Sec. II, we formulate our model. In Sec. III, we present our results for several correlation functions both below and above $T_{c}$. We conclude in Sec. IV.

\section{MODEL}

The derivation presented in this section applies for the case of temperatures below $T_{c}$. It is straightforward to adopt it for the case of temperatures above $T_{c}$.

\section{A. General formulation}

We start with the dimensionless Ginzburg-Landau functional for the superconducting energy of the island of thickness $d$, in zero applied field, 


$$
F=\frac{B_{c}(T)^{2}}{\mu_{0}} \xi(T)^{2} d \int d^{2} r\left(-|f|^{2}+\frac{1}{2}|f|^{4}+|\nabla f|^{2}\right),
$$

where integration is performed over the cross section of the nanostructure, $f$ is the dimensionless order parameter. All distances are measured in units of the temperature-dependent coherence length $\xi(T)$, and $B_{c}(T)$ is the thermodynamical critical field given by

$$
B_{c}(T)=\frac{\Phi_{0}}{2 \pi \sqrt{2} \xi(T) \lambda(T)} .
$$

We consider ultrathin islands, with $d \ll \xi(T)$ so that the problem is two dimensional. The boundary condition for the order parameter at each of the three edges of a sample is taken in its usual form for the case of a superconductor/vacuum interface,

$$
\frac{\partial f}{\partial \mathbf{n}}=0,
$$

where $\mathbf{n}$ is the unit vector in the direction perpendicular to the edge.

Let us now estimate the ratio $G(T)$ of the energy needed to suppress the order parameter to zero within the volume $\sim \xi(T)^{2} d$ and the thermal energy $k_{B} T$, using realistic values for all parameters for typical ultrathin nanostructures made of $\mathrm{Pb}$ that have been realized in recent experiments. We define $G(T)$ as

$$
G(T)=\frac{B_{c}(T)^{2} \xi(T)^{2} d}{2 \mu_{0} k_{B} T} .
$$

For the penetration depth, we use the usual expression ${ }^{10}$ for dirty superconductors, $\quad \lambda(T) \simeq 0.62 \lambda_{0} \sqrt{\frac{\dot{\xi}_{0} / l}{1-T / T_{c}}} \quad$ where $\lambda_{0} \simeq 40 \mathrm{~nm}$ is the penetration depth in bulk $\mathrm{Pb}, l \approx 2 d$ is the quasiparticle mean-free part, and $T_{c}=7.2 \mathrm{~K}$. Then, for a film of thickness $2 \mathrm{~nm}$ (similar to Ref. 5 and nearly three times smaller than in Ref. 4), operated at $T=0.75 T_{c}$, we estimate $G(T) \sim 10$.

\section{B. Fluctuative modes}

Next, we expand the order parameter $f$ around its mean value in the absence of fluctuations,

$$
f=1+\delta f .
$$

After substituting Eq. (5) into Eq. (1), we obtain the expression for the increase in superconducting energy due to thermal fluctuations,

$$
\delta F=\frac{B_{c}(T)^{2}}{\mu_{0}} \xi(T)^{2} d \int d^{2} r\left(|\delta f|^{2}+\frac{1}{2}\left(\delta f^{2}+\delta f^{* 2}\right)+|\nabla \delta f|^{2}\right),
$$

where we have kept only terms up to quadratic ones in $\delta f$ and $\delta f^{*}$.

Obviously, $\delta f$ can be represented as a superposition of plane waves. However, $\delta f$ for each fluctuative mode must satisfy the boundary condition given by Eq. (3), which is a

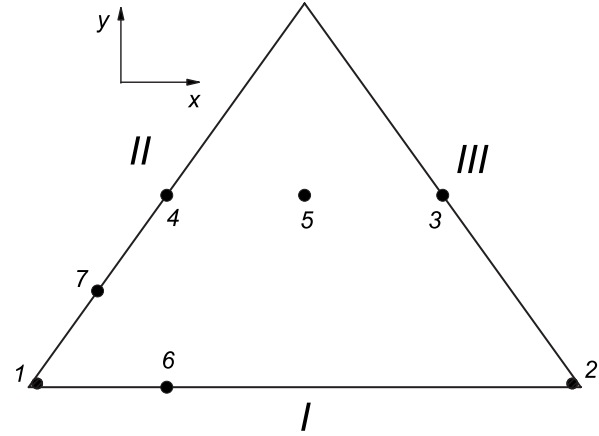

FIG. 1. Schematic image of a triangular structure. Numbers depict points, the correlation functions being calculated along the straight lines connecting these points (see in the text).

very strong restriction imposed by the geometry of our problem. In order to circumvent this difficulty, we will use, with certain modifications, an approach applied very recently in Ref. 11 for the triangular dot of graphene, for which exact electronic wave functions were found. The major difference with the graphene dot problem is in the boundary condition, which is vanishing of the wave function at the triangle's border, instead of the vanishing of its first derivative [Eq. (3)]. Following Ref. 11, as a first step we focus on the sector, confined between edges I and II [see Fig. 1] and we find the form of $\delta f$, which satisfies boundary condition [Eq. (3)] at these two edges. Consider a plane wave with the wave vector $\mathbf{k}_{1}=\left(k_{x}, k_{y}\right)$,

$$
\psi_{1}=\exp \left(-i \mathbf{k}_{1} \mathbf{r}\right)
$$

When reflected from edge $\mathrm{I}$, it is converted into wave $\psi_{2}$ with $\mathbf{k}_{2}=\left(k_{x},-k_{y}\right)$. It is then easy to see that the sum of $\psi_{1}$ and $\psi_{2}$ satisfies Eq. (3) at the edge I. This is in contrast with Ref. 11, where the difference between $\psi_{1}$ and $\psi_{2}$ was taken due to the different boundary condition. When these two waves are reflected from edge II, two new waves appear with $\mathbf{k}_{5}=-\frac{1}{2}\left(k_{x}+\sqrt{3} k_{y},-\sqrt{3} k_{x}+k_{y}\right)$ and $\mathbf{k}_{6}=-\frac{1}{2}\left(k_{x}-\sqrt{3} k_{y},-\sqrt{3} k_{x}-k_{y}\right)$. After the reflection from edge I, these two ones give rise to two more waves with $\mathbf{k}_{3}=-\frac{1}{2}\left(k_{x}-\sqrt{3} k_{y}, \sqrt{3} k_{x}+k_{y}\right)$ and $\mathbf{k}_{4}=-\frac{1}{2}\left(k_{x}+\sqrt{3} k_{y}, \sqrt{3} k_{x}-k_{y}\right)$. Finally, after reflecting from edge I, these last waves do not lead to any new waves. Then, the sum of the six wave functions $\psi_{1}+\cdots+\psi_{6}$ satisfies the boundary condition, given by Eq. (3), both at edges I and II, whatever $\mathbf{k}_{1}$ is. The same boundary condition at edge III leads to quantization rules for $\left(k_{x}, k_{y}\right)$, which differ from those found in Ref. 11. It is easy to see that the allowed $\left(k_{x}, k_{y}\right)$ split into two branches,

$$
\begin{gathered}
k_{x}^{(1)}=\frac{4 \pi}{3 L} m, \\
k_{y}^{(1)}=\frac{4 \pi}{3 L} n \sqrt{3},
\end{gathered}
$$

and

$$
k_{x}^{(2)}=\frac{4 \pi}{3 L}\left(m+\frac{1}{2}\right) \text {, }
$$




$$
k_{y}^{(2)}=\frac{4 \pi}{3 L}\left(n+\frac{1}{2}\right) \sqrt{3},
$$

where $L$ is the dimensionless length of the triangle's edge while $m$ and $n$ are arbitrary integer numbers.

For these two branches, eigenenergies are given by

$$
\begin{gathered}
E_{m n}^{(1)}=\left(\frac{4 \pi}{3 L}\right)^{2}\left(m^{2}+3 n^{2}\right), \\
E_{m n}^{(2)}=\left(\frac{4 \pi}{3 L}\right)^{2}\left[\left(m+\frac{1}{2}\right)^{2}+3\left(n+\frac{1}{2}\right)^{2}\right] .
\end{gathered}
$$

Wave functions corresponding to different $(m, n)$ but the same branch is not necessarily unique. By a proper choice of $m$ and $n$, we obtain the following basis of normalized and unique wave functions:

$$
\begin{gathered}
\varphi_{m n}^{(1)}=\frac{1}{\sqrt{6}}\left(\psi_{1}^{(1)}+\cdots+\psi_{6}^{(1)}\right), \quad n>|m|, \\
\varphi_{m n}^{(1)}=1, \quad n=m=0,
\end{gathered}
$$

where $\psi_{1}^{(1)}, \ldots, \psi_{6}^{(1)}$ depend on $\left(k_{x}^{(1)}, k_{y}^{(1)}\right)$, and

$$
\varphi_{m n}^{(2)}=\frac{1}{\sqrt{6}}\left(\psi_{1}^{(2)}+\cdots+\psi_{6}^{(2)}\right), \quad n>|m+1 / 2|,
$$

where $\psi_{1}^{(2)}, \ldots, \psi_{6}^{(2)}$ depend on $\left(k_{x}^{(2)}, k_{y}^{(2)}\right)$.

We can expand the fluctuating contribution to the order parameter in the constructed basis,

$$
\delta f=\sum_{n, m, \alpha} c_{m n}^{(\alpha)} \varphi_{m n}^{(\alpha)},
$$

where $\alpha=1,2$ stand for the two branches. We now substitute expansion (17) in Eq. (6). After performing the integration over the triangle's area, we arrive at the following expression for the statistical sum:

$$
\begin{aligned}
Z= & \prod_{n, m, \alpha} \int d\left[\operatorname{Re}\left(c_{m n}^{(\alpha)}\right)\right] d\left[\operatorname{Im}\left(c_{m n}^{(\alpha)}\right)\right] \\
& \times \exp \left\{-G(T) \frac{L^{2} \sqrt{3}}{4}\left\{\left[\operatorname{Re}\left(c_{m n}^{(\alpha)}\right)\right]^{2}\left[E_{m n}^{(\alpha)}+2\right]\right.\right. \\
& \left.\left.+\left[\operatorname{Im}\left(c_{m n}^{(\alpha)}\right)\right]^{2} E_{m n}^{(\alpha)}\right\}\right\} .
\end{aligned}
$$

It contains a product of Gaussian integrals, which can be easily evaluated analytically as

$$
Z=\prod_{n, m, \alpha} \frac{2 \pi}{L \sqrt{E_{m n}^{(\alpha)}\left(E_{m n}^{(\alpha)}+2\right) G(T) \sqrt{3}}} .
$$

\section{Correlation functions}

In order to study the spatial localization of fluctuations in the triangle below $T_{c}$, we analyze separately phase and density fluctuations since they behave in different ways. Namely, these are fluctuations of the phase of the order parameter which are responsible for the loss of the long-range order. ${ }^{12}$

It is straightforward to see, from Eq. (5), that the phase $\chi$ of the order parameter can be expressed as

$$
\chi=\frac{1}{2 i}\left(\delta f-\delta f^{*}\right) .
$$

The quantity we are interested in is the correlation function,

$$
K_{p h}\left(\mathbf{r}_{1}, \mathbf{r}_{2}\right)=\left\langle\left[\chi\left(\mathbf{r}_{1}\right)-\chi\left(\mathbf{r}_{2}\right)\right]^{2}\right\rangle_{T} .
$$

In an infinite isotropic system, this quantity depends only on the distance $\left|\mathbf{r}_{2}-\mathbf{r}_{1}\right|$. It diverges logarithmically at large distances for two-dimensional systems, expressing the loss of long-range order. However for a confined system with anisotropic geometry, $K_{p h}\left(\mathbf{r}_{1}, \mathbf{r}_{2}\right)$ depends on both $\mathbf{r}_{1}$ and $\mathbf{r}_{2}-\mathbf{r}_{1}$.

Using Eqs. (17) and (19), we arrive at the following expression for $K_{p h}\left(\mathbf{r}_{1}, \mathbf{r}_{2}\right)$ :

$$
\begin{aligned}
K_{p h}\left(\mathbf{r}_{1}, \mathbf{r}_{2}\right)= & \frac{1}{2} \sum_{m, n, \alpha}\left\langle\left[\operatorname{Im}\left(c_{m n}^{(\alpha)}\right)\right]^{2}\right\rangle_{T}\left\{\operatorname{Re}\left[\left[\Gamma_{m n}^{(\alpha)}\left(\mathbf{r}_{1}, \mathbf{r}_{2}\right)\right]^{2}\right]\right. \\
& \left.+\left|\Gamma_{m n}^{(\alpha)}\left(\mathbf{r}_{1}, \mathbf{r}_{2}\right)\right|^{2}\right\}-\frac{1}{2} \sum_{m, n, \alpha}\left\langle\left[\operatorname{Re}\left(c_{m n}^{(\alpha)}\right)\right]^{2}\right\rangle_{T} \\
& \times\left\{\operatorname{Re}\left\{\left[\Gamma_{m n}^{(\alpha)}\left(\mathbf{r}_{1}, \mathbf{r}_{2}\right)\right]^{2}\right\}-\left|\Gamma_{m n}^{(\alpha)}\left(\mathbf{r}_{1}, \mathbf{r}_{2}\right)\right|^{2}\right\},
\end{aligned}
$$

where

$$
\Gamma_{m n}^{(\alpha)}\left(\mathbf{r}_{1}, \mathbf{r}_{2}\right)=\varphi_{m n}^{(\alpha)}\left(\mathbf{r}_{1}\right)-\varphi_{m n}^{(\alpha)}\left(\mathbf{r}_{2}\right) .
$$

Thermally averaged $\left[\operatorname{Re}\left(c_{m n}^{(\alpha)}\right)\right]^{2}$ and $\left[\operatorname{Im}\left(c_{m n}^{(\alpha)}\right)\right]^{2}$ can be found analytically as

$$
\begin{gathered}
\left\langle\left[\operatorname{Re}\left(c_{m n}^{(\alpha)}\right)\right]^{2}\right\rangle_{T}=\frac{2}{G(T) L^{2} \sqrt{3}\left[E_{m n}^{(\alpha)}+2\right]}, \\
\left\langle\left[\operatorname{Im}\left(c_{m n}^{(\alpha)}\right)\right]^{2}\right\rangle_{T}=\frac{2}{G(T) L^{2} \sqrt{3} E_{m n}^{(\alpha)}} .
\end{gathered}
$$

By substituting Eqs. (23) and (24) into Eq. (21) and performing the summations, we calculate $K_{p h}\left(\mathbf{r}_{1}, \mathbf{r}_{2}\right)$ along various lines inside the triangle. This sum, however, is divergent at large $m$ and $n$, and this divergency has to be cut in a standard way [see, e.g., p. 336 of Ref. 12] at wave vectors $k \sim 1 / \xi(T)$. We found that the dependence of the final results on the particular choice of the cutoff value is rather weak, within $10 \%$.

In a similar way, we can express the fluctuation part $\delta n_{s}$ of the density of superconducting electrons $|f|^{2}$ in terms of $\delta f$ and $\delta f^{*}$,

$$
\delta n_{s}=\delta f+\delta f^{*} .
$$

By using the developed approach, it is also possible to find the density-density correlation function, defined as

$$
K_{d e n}\left(\mathbf{r}_{1}, \mathbf{r}_{2}\right)=\left\langle\delta n_{s}\left(\mathbf{r}_{1}\right) \delta n_{s}\left(\mathbf{r}_{2}\right)\right\rangle_{T} .
$$

Thermal fluctuations of the order parameter also occur at temperatures above $T_{c}$. In this case, the equilibrium order parameter is equal to zero, which leads to changes in Eqs. (5) and (18) and therefore also in Eqs. (23) and (24). We omit the derivation and we will present our final results in Sec. III 


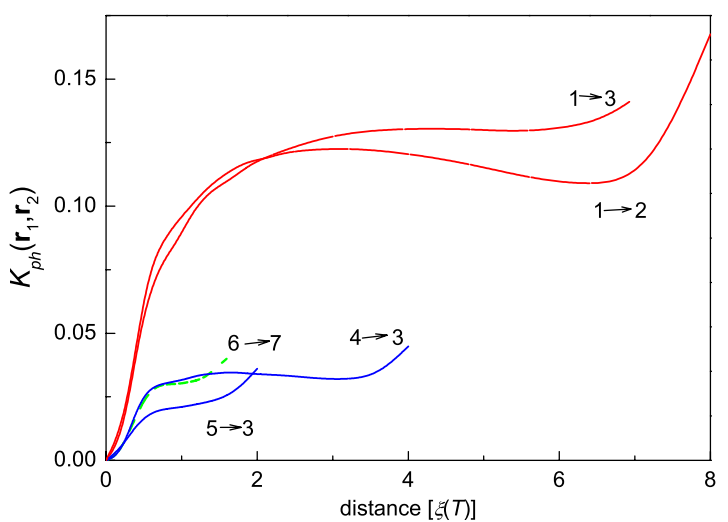

FIG. 2. (Color online) Dependence of the correlation function $K_{p h}\left(\mathbf{r}_{1}, \mathbf{r}_{2}\right)$ on $\left|\mathbf{r}_{2}-\mathbf{r}_{1}\right|$ along different paths in the triangular structure below $T_{c}$. Red lines show $K_{p h}\left(\mathbf{r}_{1}, \mathbf{r}_{2}\right)$ along the paths, which originate from the corner. Blue lines correspond to paths in the interior of the structure. Green dashed line corresponds to the path across the bisector.

for the correlation function for the order parameter itself, without separating the density and the phase

$$
K\left(\mathbf{r}_{1}, \mathbf{r}_{2}\right)=\left\langle f\left(\mathbf{r}_{1}\right)^{*} f\left(\mathbf{r}_{2}\right)\right\rangle_{T} .
$$

Above $T_{c}$, it is also convenient to normalize all the distances by $\xi(T)$, where $\xi(T)=\sqrt{\hbar^{2} / 2 m|\alpha(T)|}$.

\section{RESULTS AND DISCUSSION}

In this section, we discuss the behavior of the correlation functions in the triangle both below and above $T_{c}$.

\section{A. Temperatures below $T_{c}$}

Our results for the dependence of the phase correlation function $K_{p h}\left(\mathbf{r}_{1}, \mathbf{r}_{2}\right)$ on $\left|\mathbf{r}_{2}-\mathbf{r}_{1}\right|$ along different paths are shown in Fig. 2 for a triangle with $L=8$ [several hundreds of nanometers for the case of a nanostructure made of $\mathrm{Pb}$ at $\left.T=0.75 T_{c}, G\left(T=0.75 T_{c}\right)=10\right]$. Different curves show the behavior of $K_{p h}\left(\mathbf{r}_{1}, \mathbf{r}_{2}\right)$ along straight lines connecting various points inside the triangle, which are depicted in Fig. 1. Curve $1 \rightarrow 2$ corresponds to the path between the two corners of the structure. Curve $1 \rightarrow 3$ gives $K_{p h}\left(\mathbf{r}_{1}, \mathbf{r}_{2}\right)$ along the bisector starting from the corner. Curves $4 \rightarrow 3$ and $5 \rightarrow 3$ correspond to lines in the interior region of the structure [lengths of segments $1-4$ and $2-3$ are $L / 2$ while lengths of segments 4-5 and 5-3 are the same]. Curve $6 \rightarrow 7$ shows $K_{p h}\left(\mathbf{r}_{1}, \mathbf{r}_{2}\right)$ across the bisector [lengths of segments $1-6$ and 1-7 are $L / 5]$.

The shape of all these curves is similar and it generally resembles the behavior of the same correlation function for an isotropic infinite two-dimensional system. Namely, it first grows rapidly with increasing $\left|\mathbf{r}_{2}-\mathbf{r}_{1}\right|$ and then starts to demonstrate a smoother behavior. There are, however, obvious differences between the curves, both quantitative and qualitative. First, it is seen that the initial increase in $K_{p h}\left(\mathbf{r}_{1}, \mathbf{r}_{2}\right)$ is much larger [several times] for those paths which originate from the corner [curves $1 \rightarrow 2$ and $1 \rightarrow 3$ ], where, according to our initial guess, fluctuations are stronger. Thus, we can

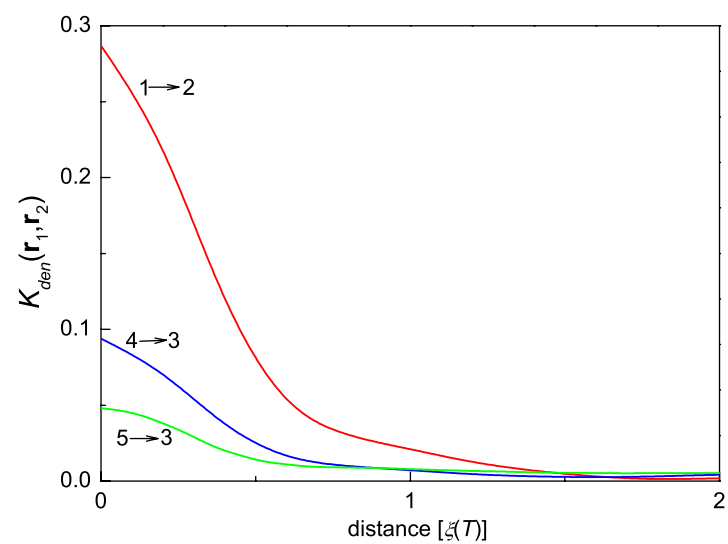

FIG. 3. (Color online) Dependence of the correlation function $K_{\text {den }}\left(\mathbf{r}_{1}, \mathbf{r}_{2}\right)$ on $\left|\mathbf{r}_{2}-\mathbf{r}_{1}\right|$ along different paths within the triangular structure below $T_{c}$. Red line shows $K_{d e n}\left(\mathbf{r}_{1}, \mathbf{r}_{2}\right)$ along the path, which originates from the corner. Blue line corresponds to path, which starts from the edge toward the interior of the structure. Green line shows $K_{d e n}\left(\mathbf{r}_{1}, \mathbf{r}_{2}\right)$ starting from the interior to the edge.

conclude that this guess is justified. Second, $K_{p h}\left(\mathbf{r}_{1}, \mathbf{r}_{2}\right)$ along the curve $1 \rightarrow 2$ starts to rapidly increase again, when approaching the other corner, while such an increase for other curves is not so pronounced. This feature implies that the order parameter between different corners is more decoherent, which again supports our hypothesis. For the range of parameters used here, we see that the maximum $\sqrt{K_{p h}\left(\mathbf{r}_{1}, \mathbf{r}_{2}\right)}$ is nearly 0.4 , which implies that the coherence between the order parameter inside different corners starts to be lost.

Figure 3 gives the density-density correlation function $K_{d e n}\left(\mathbf{r}_{1}, \mathbf{r}_{2}\right)$ for three directions, along paths $1 \rightarrow 2,4 \rightarrow 3$, and $5 \rightarrow 3$. We again see that the correlation function in the corner $[1 \rightarrow 2]$ is much larger than the same function in the interior $[4 \rightarrow 3$ and $5 \rightarrow 3]$. This shows that not only the phase of the order parameter but also the density of superconducting electrons fluctuates stronger in the corners. It is also of interest to note that the comparison of $K_{d e n}\left(\mathbf{r}_{1}, \mathbf{r}_{2}\right)$ along paths $4 \rightarrow 3$ and $5 \rightarrow 3$ shows that fluctuations are stronger near the triangle's surface as compared to the central part of the nanostructure.

The strength of thermal fluctuations at the given point $\mathbf{r}_{1}$ may be characterized by the density-density correlation function $K_{d e n}\left(\mathbf{r}_{1}, \mathbf{r}_{2}\right)$ with $\left|\mathbf{r}_{2}-\mathbf{r}_{1}\right| \sim \xi(T)$ averaged over all such values of $\mathbf{r}_{2} \cdot{ }^{12,13}$ Although $K_{d e n}\left(\mathbf{r}_{1}, \mathbf{r}_{2}\right)$ is usually peaked within nearly $1.5 \xi(T)$ in the corners for various triangle's sizes, such an averaging implies that the typical width of the geometry-induced fluctuating region in each of the three corners is broader: we estimate it to be several $\xi(T)$.

In order to visualize the effect of geometry on thermal fluctuations, we construct a "phase diagram" for the case of a triangular $\mathrm{Pb}$ nanostructure with the edge length $15 \xi(0)$ and thickness $d=5 \mathrm{~nm}$. We take $1 \rightarrow 3$ path [along the bisector] and for each point $\mathbf{r}$ at this path we calculate $\sqrt{K_{d e n}(\mathbf{r}, \mathbf{r})}$, which shows the average fluctuation of the density of superconducting electrons. We have chosen the following qualitative criterion: if this quantity exceeds 0.15 at a certain temperature, we enter the fluctuative regime. Our results for the crossover between low-temperature and fluctuative regimes 


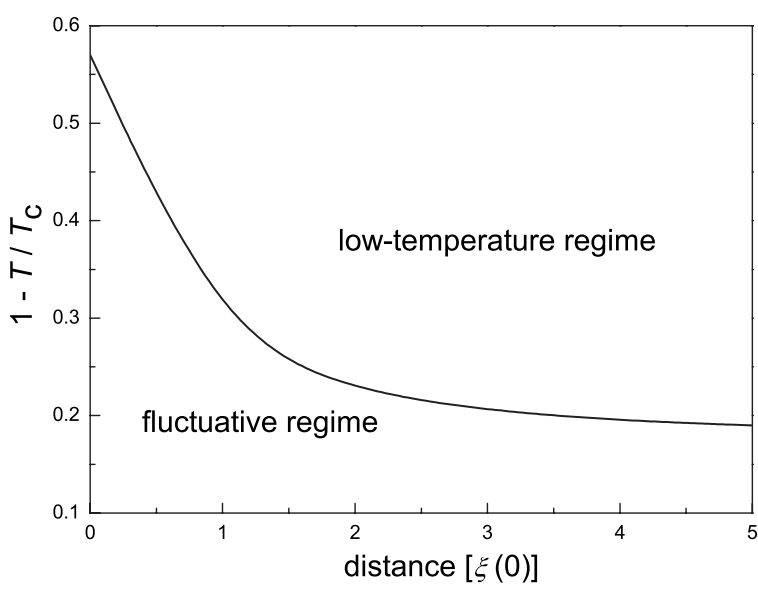

FIG. 4. Phase diagram of the triangular structure below $T_{c}$ indicating the region where fluctuations are more pronounced.

are presented in Fig. 4 in the plane of the distance along the $1 \rightarrow 3$ path and $1-T / T_{c}$. We indeed see that the characteristic temperature for the crossover in the corners strongly depends on the position. Of course, the transition between the two domains in Fig. 4 is not abrupt. It is more appropriate to talk of different regimes, which are separated by a crossover region. Thus, the suggested picture is very qualitative and it is given for illustrative purposes to supplement an idea we want to convey.

\section{B. Temperatures above $T_{c}$}

The order-parameter correlation function $K\left(\mathbf{r}_{1}, \mathbf{r}_{2}\right)$ above $T_{c}$, defined in Eq. (27), has to decrease ${ }^{12}$ with increasing $\left|\mathbf{r}_{2}-\mathbf{r}_{1}\right|$. The results of our calculations for $K\left(\mathbf{r}_{1}, \mathbf{r}_{2}\right)$ along different paths are presented in Fig. 5, where the correlation function is plotted for $T=1.25 T_{c}$ for the triangle with length $L=8$ [in terms of $\xi(T)] . K\left(\mathbf{r}_{1}, \mathbf{r}_{2}\right)$ is normalized by its value

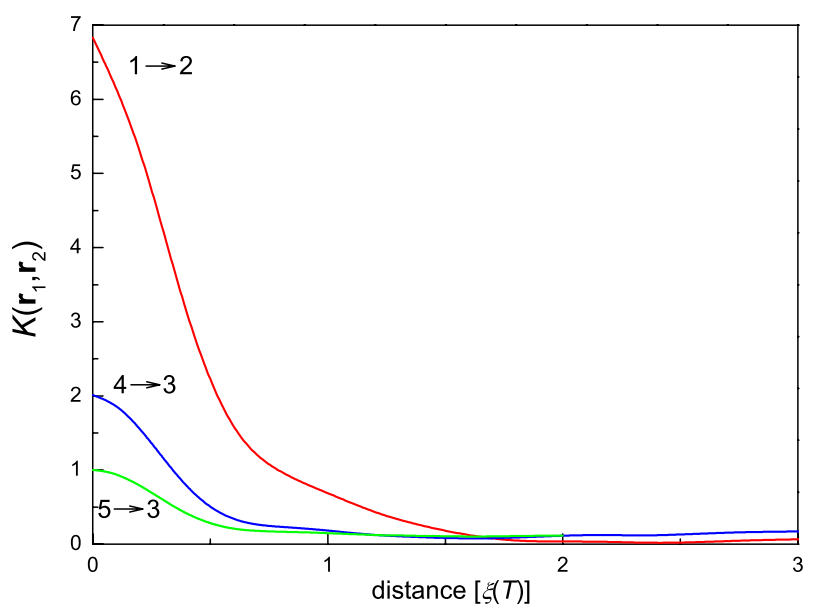

FIG. 5. (Color online) Dependence of the correlation function $K\left(\mathbf{r}_{1}, \mathbf{r}_{2}\right)$ on $\left|\mathbf{r}_{2}-\mathbf{r}_{1}\right|$ along different paths within the triangular structure above $T_{c}$. Red line shows $K\left(\mathbf{r}_{1}, \mathbf{r}_{2}\right)$ along the path, which originates from the corner. Blue line corresponds to path, which starts from the edge toward the interior of the structure. Green line shows $K\left(\mathbf{r}_{1}, \mathbf{r}_{2}\right)$ starting from the interior to the edge.
$K(\mathbf{r}, \mathbf{r})$ in the point 5 , which is located in the central part of the nanostructure (bulk). We indeed see the expected rapid decay of $K\left(\mathbf{r}_{1}, \mathbf{r}_{2}\right)$ with increasing $\left|\mathbf{r}_{2}-\mathbf{r}_{1}\right|$ but again the correlation function calculated along the path, which originates from the corner $[1 \rightarrow 2]$, is rather different from those, which correspond to lines inside the interior of the structure. Namely, the average value of the density of superconducting electrons induced by thermal fluctuations is much larger in the corners. Fluctuations near the surface are stronger than far from the surface [curves $4 \rightarrow 3$ and $5 \rightarrow 3$ ]. The width of the geometry-enhanced fluctuating region in the corners is again estimated to be several $\xi(T)$.

The comparison of our results for the correlation functions below and above $T_{c}$ shows that superconductivity is getting suppressed, below $T_{c}$, starting from the corners, while, above $T_{c}$, the order parameter preferentially nucleates again in the corners. However, there is no contradiction since if we consider a triangle with fixed sizes and if we start to increase $T$ toward $T_{c}, \xi(T)$ becomes infinitely large at $T_{c}$. This means that, close enough to $T_{c}$ on both sides of the transition, the triangle is actually in the zero-dimensional regime so that the order parameter does not vary in space. In other words, the fluctuating region in the vicinity of $T_{c}$ expands from the corners to the whole structure, while by tuning temperature away from $T_{c}$, one can make thermal fluctuations stronger in the corners than in the interior. Of course, the length of the triangle's edge should be much larger than $\xi(0)$ in order to see such a spatial localization of thermal fluctuations.

The geometry-induced localization of thermal fluctuations is expected to occur in nanostructures of various shapes, not only triangular ones. The general tendency is obvious: the sharper the corner, the stronger the fluctuations in this corner. For instance, in the corners of square-shaped samples, the width of the fluctuative region can be expected to be much smaller than in the corners of the triangular structures.

\section{CONCLUSION}

We have studied thermal fluctuations of the order parameter in quasi-two-dimensional superconducting structures of triangular shape. We considered the case of zero applied field both below and above $T_{c}$. It was shown that the order parameter exhibits much larger fluctuations in the corners of the structure and the width of such a geometry-induced fluctuating region is several $\xi(T)$. This unusual behavior can be attributed to the fact that the confinement of the condensate in the corners lowers locally the effective dimensionality of the system, thus making thermal fluctuations more pronounced. The condensate in the corners can serve as a source of thermal noise in superconducting devices based on ultrathin nanostructures and microstructures.

\section{ACKNOWLEDGMENTS}

This work was supported by the "Odysseus" Program of the Flemish government, FWO-Vl, and the Belgian Science Policy (IAP). W.V.P. acknowledges supports from the RFBR [Project No. 09-02-00248] and the "Dynasty Foundation." 
${ }^{1}$ T. Zhang, P. Cheng, W.-J. Li, Y.-J. Sun, G. Wang, X.-G. Zhu, K. He, L. Wang, X. Ma, X. Chen, Y. Wang, Y. Liu, H.-Q. Lin, J.-F. Jia, and Q.-K. Xue, Nat. Phys. 6, 104 (2010).

${ }^{2}$ A. K. Geim, S. V. Dubonos, I. V. Grigorieva, K. S. Novoselov, F. M. Peeters, and V. A. Schweigert, Nature (London) 407, 55 (2000).

${ }^{3}$ H. Bartolf, A. Engel, A. Schilling, K. Il'in, M. Siegel, H.-W. Hubers, and A. Semenov, Phys. Rev. B 81, 024502 (2010).

${ }^{4}$ T. Cren, D. Fokin, F. Debontridder, V. Dubost, and D. Roditchev, Phys. Rev. Lett. 102, 127005 (2009).

${ }^{5}$ T. Nishio, T. An, A. Nomura, K. Miyachi, T. Eguchi, H. Sakata, S. Lin, N. Hayashi, N. Nakai, M. Machida, and Y. Hasegawa, Phys. Rev. Lett. 101, 167001 (2008).

${ }^{6}$ W. V. Pogosov, Phys. Rev. B 81, 184517 (2010).

${ }^{7}$ A. D. Hernández, B. J. Baelus, D. Domínguez, and F. M. Peeters,
Phys. Rev. B 71, 214524 (2005); J. R. Kirtley, C. C. Tsuei, V. G. Kogan, J. R. Clem, H. Raffy, and Z. Z. Li, ibid. 68, 214505 (2003).

${ }^{8}$ L. F. Chibotaru, A. Ceulemans, V. Bryndoncx, and V. V. Moshchalkov, Nature (London) 408, 833 (2000).

${ }^{9}$ V. R. Misko, V. M. Fomin, J. T. Devreese, and V. V. Moshchalkov, Phys. Rev. Lett. 90, 147003 (2003).

${ }^{10}$ M. Tinkham, Introduction to Superconductivity (Dover, New York, 2004).

${ }^{11}$ A. V. Rozhkov and F. Nori, Phys. Rev. B 81, 155401 (2010).

${ }^{12}$ A. Larkin and A. Varlamov, Theory of Fluctuations in Superconductors (Oxford University Press, Oxford, 2004).

${ }^{13} \mathrm{~V}$. V. Schmidt, in The Physics of Superconductors: Introduction to Fundamentals and Applications, edited by P. Muller and A. V. Ustinov (Springer-Verlag, Berlin, 1997). 